\title{
The 3.2A resolution structure of human mTORC2
}

\author{
Alain Scaiola ${ }^{2 \dagger}$, Francesca Mangia ${ }^{1 \dagger}$, Stefan Imseng $^{1}$, Daniel Boehringer ${ }^{3}$, Karolin \\ Berneiser $^{1}$, Mitsugu Shimobayashi ${ }^{1}$, Edward Stuttfeld ${ }^{1}$, Michael N. Hall ${ }^{1}$, \\ Nenad Ban $^{2 *}, \&$ Timm Maier ${ }^{1 *}$
}

${ }^{1}$ Biozentrum, University of Basel, Klingelbergstrasse 50/70, 4056 Basel, Switzerland

${ }^{2}$ Institute for Molecular Biology and Biophysics, ETH Zürich, Zürich, Switzerland

${ }^{3}$ Cryo-EM Knowledge Hub, ETH Zurich, 8093 Zurich, Switzerland

$\dagger$ These authors contributed equally to this work

* Correspondence should be addressed to N.B. or T.M.

E-mail: ban@mol.biol.ethz.ch, timm.maier@unibas.ch 


\section{Abstract:}

The protein kinase mammalian target of rapamycin (mTOR) is the central regulator of cell growth. Aberrant mTOR signaling is linked to cancer, diabetes and neurological disorders. mTOR exerts its functions in two distinct multiprotein complexes, mTORC1 and mTORC2. Here we report a Rictor and the substrate-binding SIN1 subunits, identifies the C-terminal domain of Rictor as the source of the rapamycin insensitivity of $\mathrm{mTORC} 2$, and resolves mechanisms for $\mathrm{mTORC} 2$ regulation by complex destabilization. Two novel small molecule binding sites are visualized, an inositol hexakisphosphate (InsP6) pocket in mTOR and an mTORC2-specific nucleotide binding site in Rictor which also forms a zinc finger. Structural and biochemical analyses suggest that InsP6 and nucleotide binding do not control mTORC2 activity directly but rather have roles in folding or ternary interactions. These insights provide a firm basis for studying mTORC2 signaling and for developing mTORC2-specific inhibitors. 


\section{Main Text:}

The serine/threonine kinase mTOR, a phosphatidylinositol 3-kinase-related kinase (PIKK) (1-3), controls cell growth by balancing anabolic and catabolic metabolism $(1,4)$. mTOR is found in two separate complexes: mTOR complex 1 (mTORC1) and mTORC2 $(1,5,6)$. mTORC1 consists of mTOR, regulatory-associated protein of mTOR (Raptor), and mammalian homolog of protein lethal with sec thirteen protein 8 (mLST8) $(5,7-9)$. mTORC2 comprises mTOR, rapamycininsensitive companion of mTOR (Rictor) $(10,11)$, stress-activated map kinase-interacting protein 1 (SIN1) $(12,13)$, and $\operatorname{mLST} 8(10,11)$, and associates with the facultative subunit protein observed with Rictor-1/2 (Protor-1/2) $(14,15)$. mTORC2 is activated by insulin and phosphoinositide 3kinase (PI3K) signaling $(6,16)$ and acts on cell survival and proliferation (4) by phosphorylating the AGC family kinases: Akt, PKC and SGK (1, 4, 17-19). mTORC2 also promotes tumorigenesis via upregulation of lipid biosynthesis (20).

mTOR inhibitors played a major role in the elucidation of mTOR signaling and are used in cancer treatment $(21)$. The polyketide rapamycin specifically inhibits $\operatorname{mTORC1}(7,8)$ by forming a complex with the cellular protein FKBP12 that then binds the FKBP-rapamycin binding (FRB) domain in mTOR (Fig. 1) (22). ATP-like inhibitors target the ATP-binding site in the kinase catalytic domain of the mTORCs or the structurally related PI3K (23). Recently, mTORC2selective inhibitors were identified, but their mechanism of action remains unknown $(24,25)$. Several intermediate resolution reconstructions of (m)TOR complexes (26-30) and high-resolution reconstructions of human $\mathrm{mTORC1(31)}$ have been reported, but no high-resolution information on mTORC2 is available. Of the mTORC2 accessory proteins, only the isolated pleckstrin homology $(\mathrm{PH})$ and CRIM domains of SIN1 have been structurally characterized (32-34). For 
Rictor, fold- and secondary structure-based models have been proposed based on intermediate resolution cryo-electron microscopy (cryo-EM) reconstructions (28-30).

To investigate the structure of mTORC2 and the mechanism of its regulation, we co-expressed recombinant components of human mTORC2 (mTOR, mLST8, Rictor and SIN1) in Spodoptera frugiperda cells. The assembled complex, purified using tag-directed antibody affinity followed by size exclusion chromatography, was analyzed by cryo-EM (Fig. 1B, and Figs. S1 and S2) in the presence of ATP $\gamma \mathrm{S}$ and either the full-length substrate Akt1 (Fig. S3) or an Akt1 variant missing the PH domain ( $\triangle \mathrm{PH}-\mathrm{Akt} 1)$, or in the absence of Akt1 with and without ATP $\gamma \mathrm{S}$ (Fig. S2). The sample prepared in the presence of ATP $\gamma \mathrm{S}$ and $\triangle \mathrm{PH}-\mathrm{Akt1}$ yielded the highest overall resolution of $3.2 \AA$ (Density A in Fig. S2).

mTORC2 forms a rhomboid-shaped dimer (Fig. 1C) as observed in lower resolution mTORC2 reconstructions (28-30). The mTOR kinase forms the core of mTORC2 with mLST8 on the periphery, close to the active site cleft, similar to mTOR-mLST8 in mTORC1 $(26,31)$. In the overall reconstruction, as a consequence of EM refinement of a flexible molecule, one half of the dimer showed better local resolution (Fig. 1B and Figs. S4A-C and Movie S1). Therefore, focused refinement on a unique half of the assembly improved the resolution to $3.0 \AA$ (Density $\mathrm{C}$ in Fig. S2), and these maps were used for structural modelling (Fig. S4D-F). Previous mTORC2 and yeast TORC2 reconstructions (28-30) revealed that the two mTOR FAT domains are in closer proximity to each other than observed in mTORC1 $(26,31,35)$ and in the current structure, the distance between the mTOR FAT domains is further reduced (Fig. S5A). Irrespective of these structural differences between the two mTORCs, the catalytic site in mTORC2 closely resembles the catalytic site in mTORC1 without Rheb-mediated activation (31), suggesting that mTORC2 may be activated by a yet to be defined mechanism. 
Previous studies of mTORC2 subunits Rictor and SIN1 or their yeast orthologs were not of sufficient resolution to allow de novo model building, resulting in ambiguous or inconsistent interpretations $(28,30,36)$. Here we unambiguously model all structured regions of Rictor and the N-terminal region of SIN1 (Fig. 2A-C), whereas the middle and C-terminal part of SIN1 retain high flexibility and are not resolved. The fold of Rictor differs substantially from previous interpretations (28) (Fig. S5B-C). Rictor is composed of three interacting stacks of $\alpha$-helical repeats, here referred to as the ARM domain (AD), the HEAT-like domain (HD), and the Cterminal domain (CD) (Fig. 2A-C and Fig. S6A). The N-terminal AD (residues 26-487) forms a large superhelical arrangement of nine ARM repeats (Fig. 2A-B) that structurally separates the HD and CD. The HD (residues 526-1007), interpreted as two separate domains in previous lower resolution studies $(28,30)$, is composed of ten HEAT-like repeats. In sequence space, the HD and CD of Rictor are separated by an extended stretch of residues (1008-1559) that are predicted to be disordered (37) and are not resolved in our reconstruction. We refer to this region as the phosphorylation-site region (PR) because it contains most of Rictor's phosphorylation sites (38). The two ends of the PR are anchored by a two-stranded $\beta$-sheet at the top of the HD, which is thus termed the PR anchor (Fig. 2B-C and Fig. S6A). From here, a partially flexible linker wraps around the $\mathrm{AD}$ and the mTOR FRB domain extending toward the $\mathrm{CD}$ (Fig. 2B and Fig. S6C).

The structured parts of the CD form a four-helix bundle and a zinc finger, with bound $\mathrm{Zn}^{2+}$, in the vicinity of the Rictor N-terminus (Fig. 2A and Fig. S6B). Residues coordinating the zinc ion are highly conserved in metazoan Rictor (Fig. S6F). In earlier work, this domain had been interpreted as representing the SIN1 domain (28). The complete CD is absent in sequences of fungal Rictor orthologs, but other large extensions in yeast Rictor and SIN1 sequences may occupy the equivalent location in yeast TORC2, as observed in an intermediate resolution reconstruction of 
budding yeast TORC2(29) (Fig. S6D-E). Increased levels of $\mathrm{Zn}^{2+}$ have been reported to stimulate Akt S473 phosphorylation in cells (39-41), but no direct involvement of mTORC2 activation has been demonstrated.

Contacts between Rictor and mTOR are made by the Rictor AD, which sits between the proximal mTOR central HEAT domain and the N-terminal HEAT repeat domain, of the distal mTOR subunit (Fig. 2B). Due to its positioning on top of the mTOR FRB domain, the CD of Rictor blocks binding of FKBP12-rapamycin to mTORC2, thereby explaining mTORC2's insensitivity to rapamycin $(5,10,11,36)$ (Fig. 2D).

The SIN1 subunit of mTORC2 exhibits an unexpected structural organization. The Nterminal region (residues 2-137), contrary to earlier interpretations, does not form an independently folding domain but interacts tightly with Rictor and mLST8 in an extended conformation (Fig. 2A-C and 3A-E). The CRIM, RBD and PH domains of SIN1, however, remain flexibly disposed. The N-terminus of SIN1 is inserted into a deep cleft at the interface of the AD and HD of Rictor. The N-terminal Ala2 with a structurally resolved acetylated N-terminus, and Phe3 of SIN1 are buried in a hydrophobic pocket of Rictor (Fig. 3C,D and Fig. S7A). The anchored N-terminal region of SIN1 forms two short helices (residues 6 to 33) inserted into grooves on the surface of the Rictor AD (Fig. 3D) and then continues with a flexible sequence segment toward the Rictor CD (Figs. 2B-C and 3C and Fig. S7B). Protruding from the Rictor CD, SIN1 forms a helical segment, referred to as the "traverse", that spans the distance to mLST8 across the mTORC2 kinase cleft (Fig. 3C and Fig. S7B-C). The next region of SIN1 interacts with the fourth strand of the second blade of the mLST8 propeller by $\beta$-strand complementation, leading to displacement of an mLST8 loop relative to the structure of mLST8 in mTORC1 (Fig. 3C,E and 
Fig. S7D). SIN1 then follows the surface of the mLST8 propeller, finally forming an $\alpha$-helix anchored between the first and seventh blades of mLST8.

SIN1 integrates into the Rictor fold and connects Rictor with mLST8, suggesting a direct role in stabilizing mTORC2. To test the relevance of the anchoring of the N-terminus of SIN1 on Rictor, we extended the N-terminus of SIN1. Insertion of residues impairs critical interactions observed for the acetylated N-terminus of SIN1 and prevents Rictor integration into mTORC2, as observed in Baculovirus-mediated expression of mTOR components followed by pull-down assays (Fig. 3B and Fig. S8). Therefore, SIN1 acts as an integral part of the Rictor structure that critically stabilizes interdomain interactions, explaining the difficulties observed in purifying isolated Rictor (28).

These observations are also consistent with the locations of post-translational modifications or mutations that affect mTORC2 activity. SIN1 phosphorylation at Thr86 and Thr398 has been reported to reduce mTORC2 integrity and kinase activity toward Akt Ser473 (42). Thr86 in SIN1, which is a target for phosphorylation by S6 kinase (42), is bound to a negatively charged pocket of the Rictor CD (Fig. 3C and Fig. S7C). Phosphorylation of Thr86 would lead to repulsion from this pocket, destabilizing the interaction between Rictor and mTOR-mLST8 and presumably the entire mTORC2 assembly, in agreement with earlier in vivo and in vitro observations (42). The importance of SIN1 in connecting Rictor to mLST8 and, therefore also indirectly to mTOR, is also consistent with the requirement of mLST8 for mTORC2 integrity $(43,44)$.

A poorly resolved density linked to the SIN1 helix anchored to mLST8 is observed in all reconstructions. In previous structural studies of yeast TORC2, a similar region of density was associated with the CRIM domain of Avo1, the yeast SIN1 ortholog (29, 36). Most likely, it represents the mobile substrate-binding CRIM domain that directly follows the helix in the SIN1 
sequence and has a matching shape based on the solution structure of the S. pombe SIN1 CRIM domain (33, 34) (Fig. 3C-F and Fig. S9A,C). The positions of the SIN1 RBD and PH domains remain unresolved. In the dataset collected for samples with added full-length Akt1 (Dataset 2 in Fig. S2), we observed additional low-resolution density (Fig. 3F and Fig. S9B-C) between the hypothetic CRIM domain and Rictor AD and CD in the vicinity of the mTOR active site. This density, not of sufficient resolution to assign specific interactions, may represent parts of bound Akt1 or SIN1 domains (Fig. S9C).

A proposed regulatory mechanism for mTORC2 involves ubiquitylation of mLST8 on Lys305 and Lys313 (45). Loss of ubiquitylation by K305R and/or K313R mutation, or truncation of mLST8 at Tyr297, leads to mTORC2 hyperactivation and increased Akt phosphorylation (45). Indeed, mLST8 Lys305 is proximal to the SIN1 helix anchoring the CRIM domain. Ubiquitylation of Lys305 would prevent association of the SIN1 helix, leading to dislocation of the SIN1 CRIM domain required for substrate recruitment (Figs. 3C and 4C). Ubiquitylation of Lys313, which is found on the lower face of mLST8 (Figs. 3C and 4C), presumably also interferes with positioning of the CRIM domain (Fig. S9).

We observed two novel, small molecule binding sites outside the mTOR catalytic site, which is itself occupied by ATP $\gamma$ S. The first (A-site) (Fig. 4A and Fig. S10B) is located in the HD of Rictor and is thus specific to mTORC2. The second (I-site) (Fig. 4B and Fig. S10C) is located in the FAT domain of mTOR and is thus common to mTORC1 and mTORC2.

The density of the small molecule in the A-site matched that of an ATP molecule and was confirmed to be ATP (or ATP $\gamma \mathrm{S}$ ) through a comparison of cryoEM reconstructions of mTORC2 with and without ATP $\gamma \mathrm{S}$ added at a near physiological concentration of $2 \mathrm{mM}$ (Datasets 1 and 4, Fig. S2 and S10A). The A-site does not resemble any known ATP binding site. Positively charged 
amino acids (Lys541, Arg575, Arg576, Arg572) of the A-site are conserved in Rictor orthologs from yeast to human (Figs. S6E and S11). Other residues are not conserved, hinting at the possibility for interactions with alternative negatively charged ligands. The A-site is located approximately $100 \AA$ from the mTOR catalytic site. Ligand binding to the A-site caused neither long-range allosteric change affecting the kinase site nor local structural perturbations (Fig. S12).

To investigate the effect of ligand binding to the A-site, we generated a series of Rictor variants with a mutated A-site (Table S1). Variants with three or four mutated residues (A3 and A4) assembled into mTORC2 (Fig. S13B) while variant A5 was defective in assembly (Fig. S13B-D). Cryo-EM reconstructions of variants A3 and A4 in the presence of ATPyS (Fig. S12I-J,K-L) confirmed that the chosen mutations abolish ligand binding under near physiological conditions (Figs. S10A and S12J,L). Purified mTORC2 containing Rictor variants A3 or A4 exhibited thermal stability and kinase activity, in an Akt1 in vitro phosphorylation assay, comparable to wild-type mTORC2. (Figs. S14B and S15A,B). Complementation of a Rictor knockout (KO) in HEK293T cells by transfected Rictor-WT, or Rictor variant A3 yielded comparable levels of AKT-S473 phosphorylation (Table S1 and Fig. S16). Altogether, the above analyses indicate that ligand binding to the A-site does not directly influence mTORC2 kinase activity, suggesting rather a role in the interaction with other, yet unidentified, partner proteins of mTORC2.

The I-site is formed entirely by the FAT domain of mTOR, where a large, positively charged, pocket is lined by six lysine and two arginine residues to bind an extended ligand (Fig. 4B and Fig. S10C). The I-site was still partially occupied in our reconstruction of mTORC2 prepared without addition of exogeneous ATP $\gamma \mathrm{S}$ or other relevant ligands (Data Fig. S10A). The co-purified molecule was identified by map appearance and by ion mobility spectrometry-mass spectrometry (IMS-MS) as inositol hexakisphosphate (InsP6) (Figs. S17 and S18A-F). InsP6 binds in a region, 
which is incomplete in related PI3Ks (46), but generally conserved in members of the PIKK family of kinases (47). Indeed, InsP6 was previously reported to associate with DNA-PKcs (48). Recently, structure determination of the PIKK family pseudo-kinase SMG1 revealed InsP6 binding in a region corresponding to the I-site and led the authors to postulate a corresponding binding site in mTOR but involving both the kinase domain and FAT domain (47). InsP6 has previously been observed as a structural component of multi-subunit assemblies, including the splicesome (49) and proteasome activator complex (50), and helical repeat regions have been identified as InsP6 interaction sites $(51)$.

To investigate the function of Ins6P interaction, we purified recombinant mTORC2 containing mTOR I-site mutations (Table S1). mTOR variants with two and three mutations, I2 and I3, yielded intact mTORC2 complexes (Fig. S13A), while a variant with five mutations, I5, failed to assemble into mTORC2 (Fig. S13A,C). mTORC2 containing mTOR variants I2 and I3 displayed normal kinase activity toward Akt1 in vitro (Fig. S14A). Notably, the mutations in I2 are equivalent to those reported previously to abolish completely the kinase activity of an N-terminally truncated 'naked' mTOR fragment toward a C-terminal peptide of Akt1 (47). A possible explanation for this apparent discrepancy is provided by a reduced stability of mTORC 2 assembled using the I 2 variant (but not the I3 variant) (Fig. S15A). This destabilizing effect might be more pronounced in an mTOR fragment than in the context of an assembled mTORC2 (Fig. S15A).

To investigate a possible role of InsP6 metabolism on mTORC2 activity in HEK293T cells, we knocked down (KD) and knocked out (KO) Inositol-pentakisphosphate 2-kinase (IPPK) and Multiple inositol polyphosphate phosphatase 1 (MINPP1), respectively. The former enzyme generates InsP6 whereas the latter degrades it (Fig. S19). These manipulations of InsP6 metabolizing enzymes did not alter mTORC2 kinase activity in non-stimulated cells or in cells 
stimulated with FCS and insulin (Fig. S19A-H). These biochemical results are consistent with the observed stable binding of InsP6 to mTORC2 and suggest a role of InsP6 in mTOR folding or mTOR complex assembly, rather than as an acute transient metabolic input signal to mTORC1 or mTORC2.

Here, we describe a bona fide structure of mTORC2. We visualized how SIN1 stabilizes and tethers Rictor to the mTOR-mLST8 core. SIN1 further uses mLST8 as a platform for positioning its substrate recruiting CRIM domain, revealing a new functional role for mLST8 and rationalizing the impact of SIN1 and mLST8 modifications on mTORC2 activity. We also provide the structural basis for how the Rictor CD determines mTORC2's rapamycin insensitivity, by a mechanism different from those inferred from previous structural data $(28,30)$. We identified and functionally characterized two ligand binding sites in mTORC2. The I-site in mTOR is common to mTORC1 and 2, binds InsP6 and presumably functions in mTOR folding or assembly rather than acting as a sensor site for acute changes in cellular InsP6 concentration. The mTORC2 specific A-site of Rictor binds ATP. It doesn't affect mTORC2 activity by allostery but may be involved in linking partner protein interactions to cellular nucleotide triphosphate concentrations. Altogether, the data presented here provide a firm basis for further analysis of the function of mTORC2 and its interplay with partner proteins for controlling subcellular localization (52) and regulation of activity $(1,4$, 10, 20). Interaction sites of Rictor and mLST8 with SIN1 provide an opportunity for the development of inhibitors specific for mTORC2. 


\section{References and Notes:}

1. R. A. Saxton, D. M. Sabatini, mTOR Signaling in Growth, Metabolism, and Disease. Cell 169, 361-371 (2017).

2. S. Imseng, C. H. Aylett, T. Maier, Architecture and activation of phosphatidylinositol 3-kinase related kinases. Curr Opin Struct Biol 49, 177-189 (2018).

3. C. T. Keith, S. L. Schreiber, PIK-related kinases: DNA repair, recombination, and cell cycle checkpoints. Science 270, 50-51 (1995).

4. E. Dazert, M. N. Hall, mTOR signaling in disease. Curr Opin Cell Biol 23, 744-755 (2011).

5. R. Loewith et al., Two TOR complexes, only one of which is rapamycin sensitive, have distinct roles in cell growth control. Mol Cell 10, 457-468 (2002).

6. D. Mossmann, S. Park, M. N. Hall, mTOR signalling and cellular metabolism are mutual determinants in cancer. Nat Rev Cancer 18, 744-757 (2018).

7. K. Hara et al., Raptor, a binding partner of target of rapamycin (TOR), mediates TOR action. Cell 110, 177-189 (2002).

8. D. H. Kim et al., mTOR interacts with raptor to form a nutrient-sensitive complex that signals to the cell growth machinery. Cell 110, 163-175 (2002).

9. D. H. Kim et al., GbetaL, a positive regulator of the rapamycin-sensitive pathway required for the nutrient-sensitive interaction between raptor and mTOR. Mol Cell 11, 895-904 (2003).

10. E. Jacinto et al., Mammalian TOR complex 2 controls the actin cytoskeleton and is rapamycin insensitive. Nat Cell Biol 6, 1122-1128 (2004). 
11. D. D. Sarbassov et al., Rictor, a novel binding partner of mTOR, defines a rapamycininsensitive and raptor-independent pathway that regulates the cytoskeleton. Curr Biol 14, 1296-1302 (2004).

12. M. A. Frias et al., mSin1 is necessary for Akt/PKB phosphorylation, and its isoforms define three distinct mTORC2s. Curr Biol 16, 1865-1870 (2006).

13. Q. Yang, K. Inoki, T. Ikenoue, K. L. Guan, Identification of Sin1 as an essential TORC2 component required for complex formation and kinase activity. Genes Dev 20, 2820-2832 (2006).

14. L. R. Pearce et al., Identification of Protor as a novel Rictor-binding component of mTOR complex-2. Biochem J 405, 513-522 (2007).

15. K. Thedieck et al., PRAS40 and PRR5-like protein are new mTOR interactors that regulate apoptosis. PLoS One 2, e1217 (2007).

16. V. Zinzalla, D. Stracka, W. Oppliger, M. N. Hall, Activation of mTORC2 by association with the ribosome. Cell 144, 757-768 (2011).

15 17. J. M. Garcia-Martinez, D. R. Alessi, mTOR complex 2 (mTORC2) controls hydrophobic motif phosphorylation and activation of serum- and glucocorticoid-induced protein kinase 1 (SGK1). Biochem J 416, 375-385 (2008).

18. T. Ikenoue, K. Inoki, Q. Yang, X. Zhou, K. L. Guan, Essential function of TORC2 in PKC and Akt turn motif phosphorylation, maturation and signalling. EMBO J 27, 1919-1931 (2008). Akt/PKB by the rictor-mTOR complex. Science 307, 1098-1101 (2005). 
20. Y. Guri et al., mTORC2 Promotes Tumorigenesis via Lipid Synthesis. Cancer Cell 32, 807823 e812 (2017).

21. J. Xie, X. Wang, C. G. Proud, mTOR inhibitors in cancer therapy. F1000Res 5, (2016).

22. J. Choi, J. Chen, S. L. Schreiber, J. Clardy, Structure of the FKBP12-rapamycin complex interacting with the binding domain of human FRAP. Science 273, 239-242 (1996).

23. D. Benjamin, M. Colombi, C. Moroni, M. N. Hall, Rapamycin passes the torch: a new generation of mTOR inhibitors. Nat Rev Drug Discov 10, 868-880 (2011).

24. A. Benavides-Serrato et al., Specific blockade of Rictor-mTOR association inhibits mTORC2 activity and is cytotoxic in glioblastoma. PLoS One 12, e0176599 (2017).

25. A. Benavides-Serrato et al., Correction: Specific blockade of Rictor-mTOR association inhibits mTORC2 activity and is cytotoxic in glioblastoma. PLoS One 14, e0212160 (2019).

26. C. H. Aylett et al., Architecture of human mTOR complex 1. Science 351, 48-52 (2016).

27. D. Baretic, A. Berndt, Y. Ohashi, C. M. Johnson, R. L. Williams, Tor forms a dimer through an N-terminal helical solenoid with a complex topology. Nat Commun 7, 11016 (2016).

28. X. Chen et al., Cryo-EM structure of human mTOR complex 2. Cell Res 28, 518-528 (2018).

29. M. Karuppasamy et al., Cryo-EM structure of Saccharomyces cerevisiae target of rapamycin complex 2. Nat Commun 8, 1729 (2017).

30. E. Stuttfeld et al., Architecture of the human mTORC2 core complex. Elife 7, (2018).

31. H. Yang et al., Mechanisms of mTORC1 activation by RHEB and inhibition by PRAS40. Nature 552, 368-373 (2017). 
32. D. Pan, Y. Matsuura, Structures of the pleckstrin homology domain of Saccharomyces cerevisiae Avo1 and its human orthologue Sin1, an essential subunit of TOR complex 2. Acta Crystallogr Sect F Struct Biol Cryst Commun 68, 386-392 (2012).

33. H. Tatebe et al., Substrate specificity of TOR complex 2 is determined by a ubiquitin-fold domain of the Sin1 subunit. Elife 6, (2017).

34. K. Furuita et al., Utilization of paramagnetic relaxation enhancements for high-resolution NMR structure determination of a soluble loop-rich protein with sparse NOE distance restraints. J Biomol NMR 61, 55-64 (2015).

35. H. Yang et al., mTOR kinase structure, mechanism and regulation. Nature 497, 217-223 (2013).

36. C. Gaubitz et al., Molecular Basis of the Rapamycin Insensitivity of Target Of Rapamycin Complex 2. Mol Cell 58, 977-988 (2015).

37. L. A. Kelley, S. Mezulis, C. M. Yates, M. N. Wass, M. J. Sternberg, The Phyre2 web portal for protein modeling, prediction and analysis. Nat Protoc 10, 845-858 (2015).

15 38. C. C. Dibble, J. M. Asara, B. D. Manning, Characterization of Rictor phosphorylation sites reveals direct regulation of mTOR complex 2 by S6K1. Mol Cell Biol 29, 5657-5670 (2009).

39. S. Lee, G. Chanoit, R. McIntosh, D. A. Zvara, Z. Xu, Molecular mechanism underlying Akt activation in zinc-induced cardioprotection. Am J Physiol Heart Circ Physiol 297, H569-575 (2009).

20 40. M. Taniguchi et al., Essential role of the zinc transporter ZIP9/SLC39A9 in regulating the activations of Akt and Erk in B-cell receptor signaling pathway in DT40 cells. PLoS One 8, e58022 (2013) 
41. T. Nimmanon, S. Ziliotto, S. Morris, L. Flanagan, K. M. Taylor, Phosphorylation of zinc channel ZIP7 drives MAPK, PI3K and mTOR growth and proliferation signalling. Metallomics 9, 471-481 (2017).

42. P. Liu et al., Sin1 phosphorylation impairs mTORC2 complex integrity and inhibits downstream Akt signalling to suppress tumorigenesis. Nat Cell Biol 15, 1340-1350 (2013).

43. Y. Hwang et al., Disruption of scaffolding function of mLST8 selectively inhibits mTORC2 assembly and function and suppresses mTORC2-dependent tumor growth in vivo. Cancer Res, (2019).

44. S. Wullschleger, R. Loewith, W. Oppliger, M. N. Hall, Molecular organization of target of rapamycin complex 2. J Biol Chem 280, 30697-30704 (2005).

45. B. Wang et al., TRAF2 and OTUD7B govern a ubiquitin-dependent switch that regulates mTORC2 signalling. Nature 545, 365-369 (2017).

46. J. E. Burke, Structural Basis for Regulation of Phosphoinositide Kinases and Their Involvement in Human Disease. Mol Cell 71, 653-673 (2018).

15 47. Y. Gat et al., InsP6 binding to PIKK kinases revealed by the cryo-EM structure of an SMG1SMG8-SMG9 complex. Nat Struct Mol Biol 26, 1089-1093 (2019).

48. L. A. Hanakahi, M. Bartlet-Jones, C. Chappell, D. Pappin, S. C. West, Binding of inositol phosphate to DNA-PK and stimulation of double-strand break repair. Cell 102, 721-729 (2000).

20 49. X. Zhang et al., Structures of the human spliceosomes before and after release of the ligated exon. Cell Res 29, 274-285 (2019). 
50. A. Toste Rego, P. C. A. da Fonseca, Characterization of Fully Recombinant Human 20S and 20S-PA200 Proteasome Complexes. Mol Cell 76, 138-147 e135 (2019).

51. Z. Ouyang, G. Zheng, D. R. Tomchick, X. Luo, H. Yu, Structural Basis and IP6 Requirement for Pds5-Dependent Cohesin Dynamics. Mol Cell 62, 248-259 (2016).

52. M. Ebner, B. Sinkovics, M. Szczygiel, D. W. Ribeiro, I. Yudushkin, Localization of mTORC2 activity inside cells. J Cell Biol 216, 343-353 (2017).

53. D. J. Fitzgerald et al., Protein complex expression by using multigene baculoviral vectors. Nat Methods 3, 1021-1032 (2006).

54. M. Andjelkovic et al., Role of translocation in the activation and function of protein kinase B. J Biol Chem 272, 31515-31524 (1997).

55. I. Lucic et al., Conformational sampling of membranes by Akt controls its activation and inactivation. Proc Natl Acad Sci U S A 115, E3940-E3949 (2018).

56. F. Bossler et al., Repression of Human Papillomavirus Oncogene Expression under Hypoxia Is Mediated by PI3K/mTORC2/AKT Signaling. mBio 10, (2019).

15 57. N. E. Sanjana, O. Shalem, F. Zhang, Improved vectors and genome-wide libraries for CRISPR screening. Nat Methods 11, 783-784 (2014).

58. E. Ahrne et al., Evaluation and Improvement of Quantification Accuracy in Isobaric Mass TagBased Protein Quantification Experiments. J Proteome Res 15, 2537-2547 (2016).

59. A. I. Nesvizhskii, A. Keller, E. Kolker, R. Aebersold, A statistical model for identifying proteins by tandem mass spectrometry. Anal Chem 75, 4646-4658 (2003). 
60. B. Kastner, Purification and Electron Microscopy of Spliceosomal snRNPs. Schenkel J. (eds) RNP Particles, Splicing and Autoimmune Diseases. Springer Lab Manual., (1998).

61. W. F. Tivol, A. Briegel, G. J. Jensen, An improved cryogen for plunge freezing. Microsc Microanal 14, 375-379 (2008).

62. D. N. Mastronarde, Automated electron microscope tomography using robust prediction of specimen movements. J Struct Biol 152, 36-51 (2005).

63. S. Q. Zheng et al., MotionCor2: anisotropic correction of beam-induced motion for improved cryo-electron microscopy. Nat Methods 14, 331-332 (2017).

64. K. Zhang, Gctf: Real-time CTF determination and correction. J Struct Biol 193, 1-12 (2016). single-particle reconstructions. J Struct Biol 128, 82-97 (1999).

66. S. H. Scheres, RELION: implementation of a Bayesian approach to cryo-EM structure determination. J Struct Biol 180, 519-530 (2012).

67. A. Punjani, J. L. Rubinstein, D. J. Fleet, M. A. Brubaker, cryoSPARC: algorithms for rapid unsupervised cryo-EM structure determination. Nat Methods 14, 290-296 (2017).

68. E. F. Pettersen et al., UCSF Chimera--a visualization system for exploratory research and analysis. J Comput Chem 25, 1605-1612 (2004).

69. J. Zivanov, T. Nakane, S. H. W. Scheres, A Bayesian approach to beam-induced motion correction in cryo-EM single-particle analysis. IUCrJ 6, 5-17 (2019).

70. T. C. Terwilliger, O. V. Sobolev, P. V. Afonine, P. D. Adams, Automated map sharpening by maximization of detail and connectivity. Acta Crystallogr D Struct Biol 74, 545-559 (2018). 
71. P. D. Adams et al., PHENIX: a comprehensive Python-based system for macromolecular structure solution. Acta Crystallogr D Biol Crystallogr 66, 213-221 (2010).

72. P. Emsley, B. Lohkamp, W. G. Scott, K. Cowtan, Features and development of Coot. Acta Crystallogr D Biol Crystallogr 66, 486-501 (2010).

73. T. D. Goddard et al., UCSF ChimeraX: Meeting modern challenges in visualization and analysis. Protein Sci 27, 14-25 (2018).

74. I. S. Fernandez, X. C. Bai, G. Murshudov, S. H. Scheres, V. Ramakrishnan, Initiation of translation by cricket paralysis virus IRES requires its translocation in the ribosome. Cell 157, 823-831 (2014).

75. N. A. Baker, D. Sept, S. Joseph, M. J. Holst, J. A. McCammon, Electrostatics of nanosystems: application to microtubules and the ribosome. Proc Natl Acad Sci U S A 98, 10037-10041 (2001).

76. F. Sievers et al., Fast, scalable generation of high-quality protein multiple sequence alignments using Clustal Omega. Mol Syst Biol 7, 539 (2011).

15 77. P. Gouet, E. Courcelle, D. I. Stuart, F. Metoz, ESPript: analysis of multiple sequence alignments in PostScript. Bioinformatics 15, 305-308 (1999).

78. J. Pei, N. V. Grishin, AL2CO: calculation of positional conservation in a protein sequence alignment. Bioinformatics 17, 700-712 (2001).

Acknowledgments: We thank T. Sharpe at the Biophysics facility and A. Schmidt at the Proteomics Core Facility of Biozentrum and the sciCORE scientific computing facility, all of University of Basel. We thank M. Leibundgut for advice with model building, A. Jomaa and S. Mattei for advice on cryoEM data processing, the ETH scientific center for optical and electron 
microscopy (ScopeM), and in particular M. Peterek and P. Tittmann for technical support. We are indebted to E. Laczko and J. Hu of the Functional Genomics Center Zürich for the help with the mass spectrometry. We thank Iva Lučić and Thomas Leonard (Max F. Perutz Laboratories, Vienna) for providing (Delta-PH) Akt1 protein.; Funding: F.M. is supported by a Fellowship for

Excellence from the Biozentrum Basel International $\mathrm{PhD}$ program. This work was supported by the Swiss National Science Foundation (SNSF) via the National Center of Excellence in RNA and Disease (project funding 138262) to N. Ban and M. N. Hall and SNSF project funding 179323 and 177084 to T. Maier.; Author contributions: AS designed the experiments, prepared the sample for cryoEM, carried data processing and structure modelling. AS and DB performed data collection. FM designed the experiments, cloned Akt1, mTORC2 mutants and Rictor mutants, expressed and purified proteins, performed the activity assays and the nanoDSF measurements. ES established the mTORC2 purification procedure. SI cloned mTORC2, contributed to data analysis and manuscript preparation. MS performed the in-cell analysis of mTORC2 activity. KB and MS performed the KO/KD of MINPP1 and IPPK. AS, FM, DB, SI, NB, MNH and TM participated in the writing of the manuscript.; Competing interests: The authors declare no competing interests.; and Data and materials availability: The high resolution cryo-EM map of the half (Density C) and full-mTORC2 (Density A) has been deposited in the Electron Microscopy Data Bank as EMD- XXX and EMD-YYYY respectively, while the corresponding model are in the Protein Data Bank as PDB ID WWW and ZZZ. Additionally, the density of mTORC2 in absence of ATP $\gamma$ S (Density F), as well as the densities showing extra density (Density G and H) were deposited in the Electron Microscopy Data Bank as EMD-AAAA, EMD-BBBB, and EMDCCCC respectively. 
bioRxiv preprint doi: https://doi.org/10.1101/2020.04.10.029835; this version posted April 10, 2020. The copyright holder for this preprint (which

was not certified by peer review) is the author/funder, who has granted bioRxiv a license to display the preprint in perpetuity. It is made available under aCC-BY-NC-ND 4.0 International license.

\section{Supplementary Materials:}

Materials and Methods

Figures S1-S20

Tables S1-S2

Movies S1-S4

External Databases S1-S7 


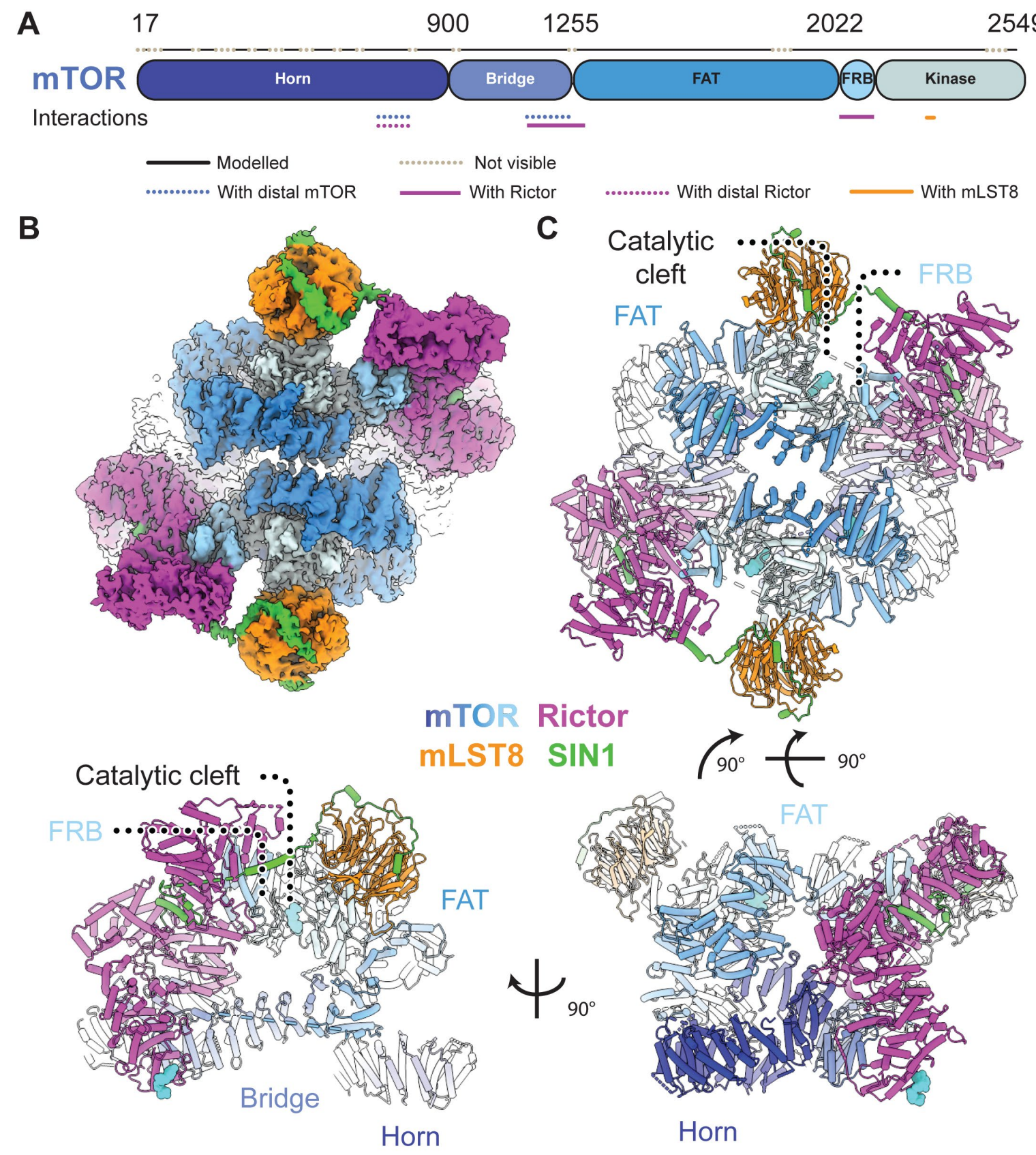

Fig. 1. Structure of mTOR complex 2 (A) Sequence-level domain organization of mTOR.

Modelled and unresolved regions are indicated as dotted lines. Interactions with other proteins in the complex are highlighted below the sequences. (B) Density of the overall cryo-EM reconstruction of mTORC2 colored according to protein subunits and mTOR domains as indicated. The top half is better resolved than the lower one, most likely due to conformational flexibility. 
bioRxiv preprint doi: https://doi.org/10.1101/2020.04.10.029835; this version posted April 10, 2020. The copyright holder for this preprint (which

was not certified by peer review) is the author/funder, who has granted bioRxiv a license to display the preprint in perpetuity. It is made available under aCC-BY-NC-ND 4.0 International license.

(C) Cartoon representation of mTORC2 in three different orientations. The proteins Rictor

(magenta) and SIN1(green) are unique to mTORC2, while mTOR (colored by domain) and mLST8 (orange) are common to both $\mathrm{mTORC} 1$ and $\mathrm{mTORC} 2$. Bound ligands are represented as cyan spheres. 
A

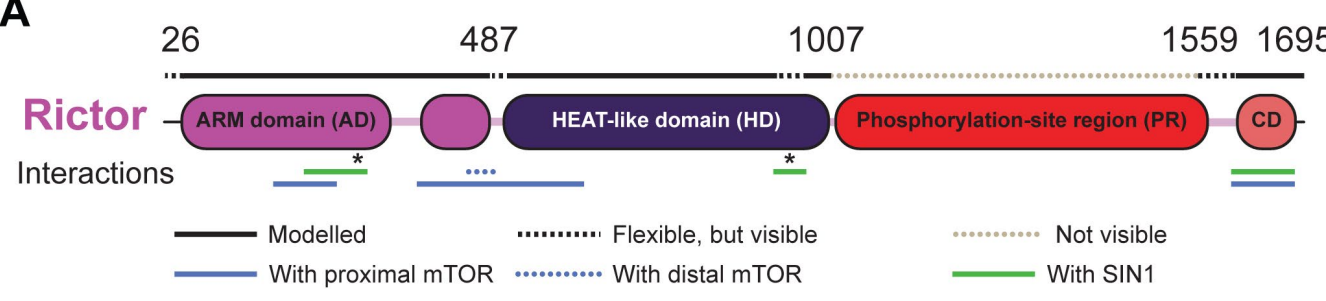

B

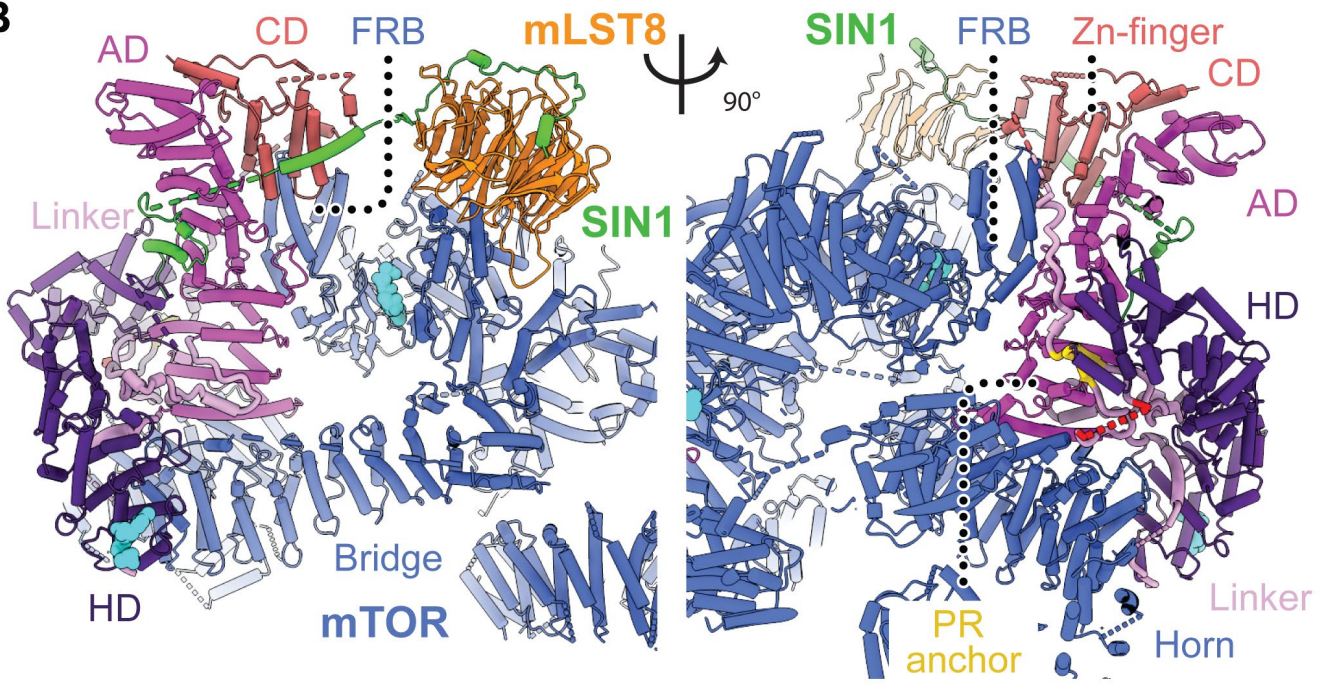

C

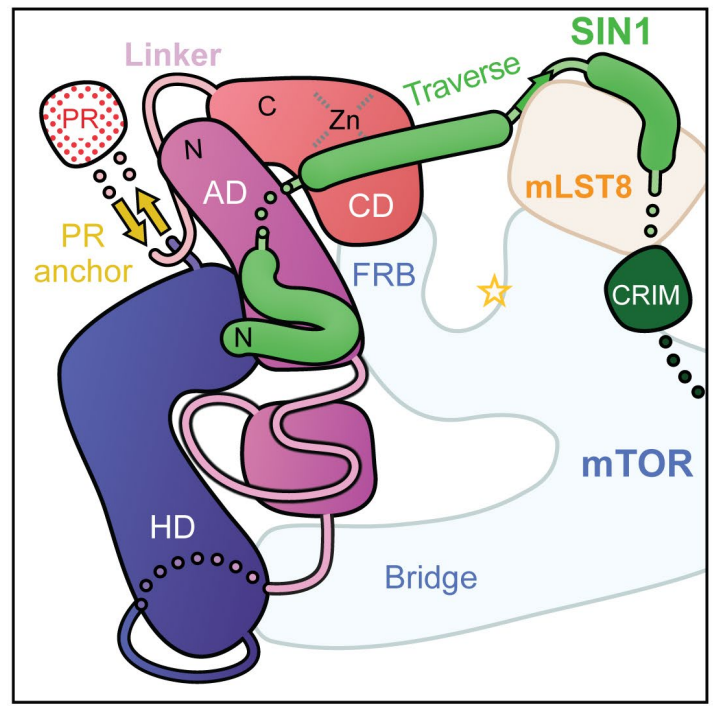

D

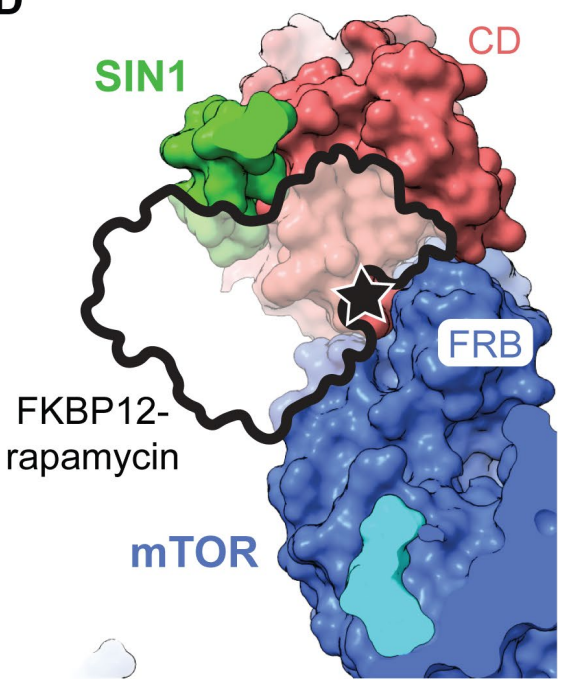

Fig. 2. The architecture of Rictor (A) Sequence-level domain organization of Rictor. Flexible and unresolved regions are indicated as dotted lines. Interactions with other proteins in the complex are highlighted below the sequences. Asterisks indicate residues interacting with the $\mathrm{N}$-terminal

region of SIN1. (B) Two views of Rictor, colored by domains. The structured part of Rictor forms three domains: an N-terminal Armadillo repeat domain (AD, magenta), a HEAT-like repeat 
domain (HD, dark magenta), and a C-terminal domain (CD, light red), the phosphorylation site region (PR) remains disordered. The sequences flanking the non-resolved PR are highlighted in red, the PR anchor is colored in gold. Bound ligands are shown as cyan spheres. (C) Schematic representation of Rictor and SIN1 domain topology. (D) The Rictor CD occupies the FRB domain and sterically blocks FKBP-rapamycin binding(26). 
A

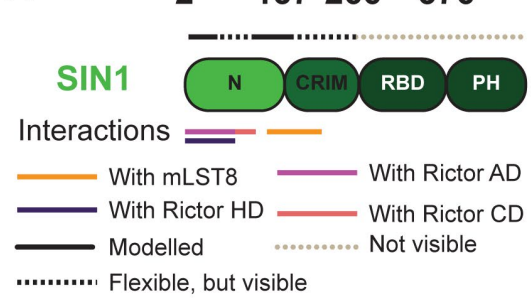

C

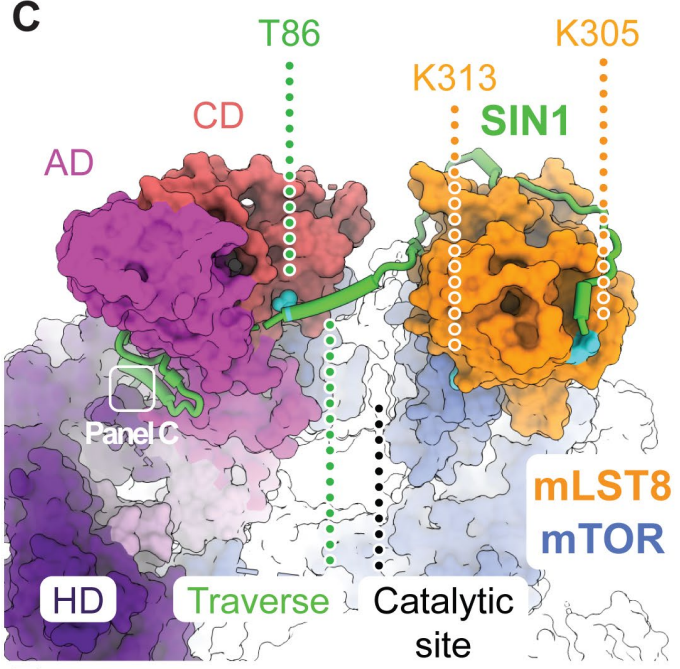

$\mathbf{E}$

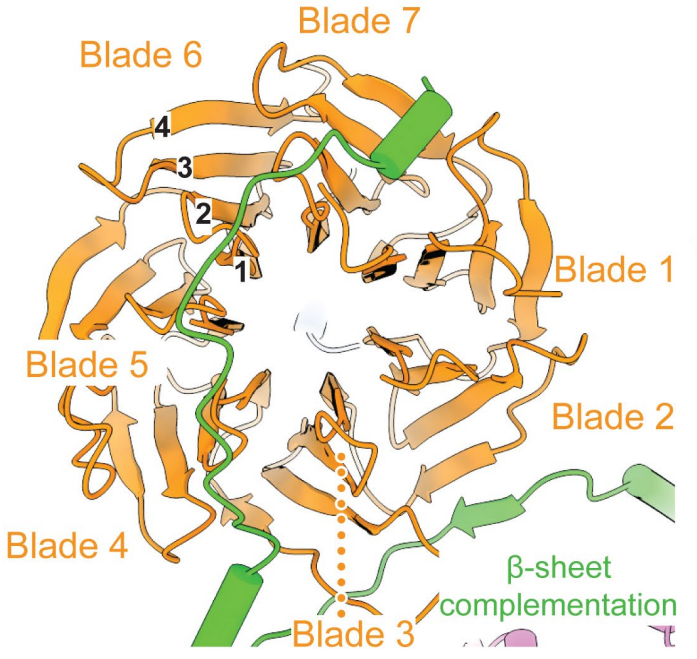

B
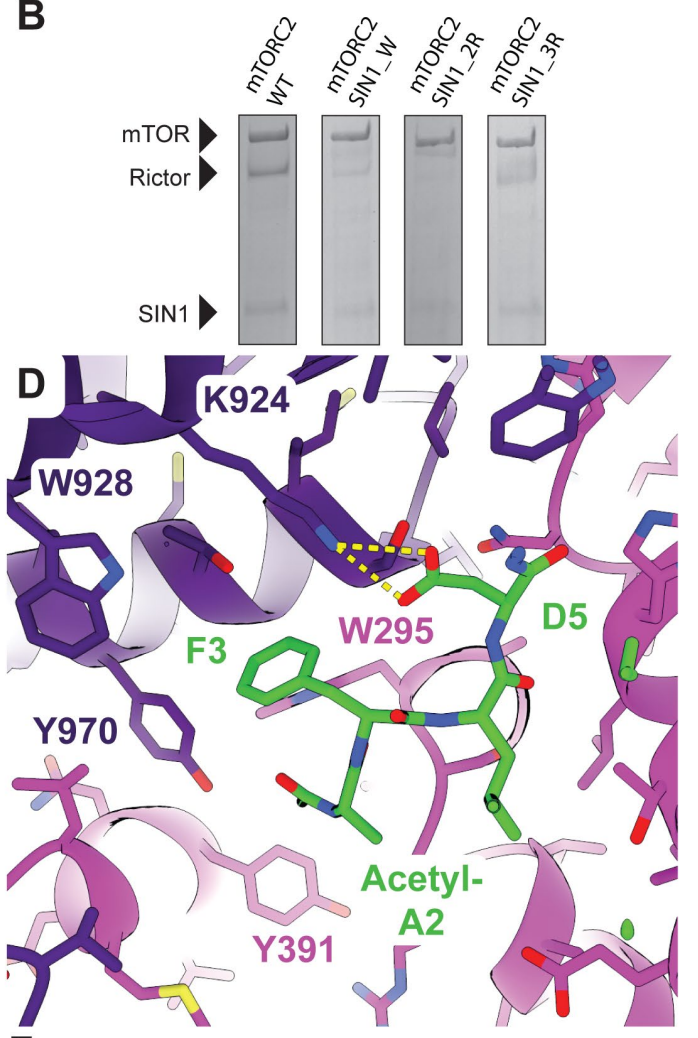

$\mathbf{F}$

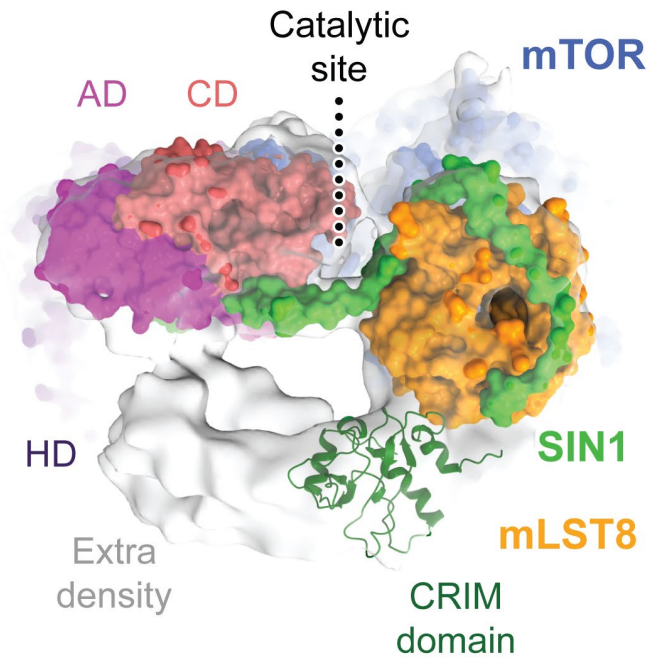

Fig. 3. The SIN1 N-terminal region is an integral component of mTORC2 (A) Sequence-level domain organization of SIN1. Flexible and unresolved regions are shown above each domain representation as dotted lines in two colors as indicated. Interactions with other proteins in the complex are indicated below the domain representation. (B) Extension of the processed SIN1 Nterminus disrupts assembly of Rictor and SIN1 with mTOR/mLST8 into mTORC2. SDS- 
polyacrylamide gel of a FLAG bead pulldown from lysates of insect cells expressing mTORC2 comprising SIN1 variants. Levels of Rictor are drastically reduced in the mTOR-based pulldown for mTORC2 carrying variants of SIN1 N-terminally extended by a tryptophan (mTORC2 SIN1_W), two consecutive arginines (mTORC2 SIN1_2R) and three consecutive arginines (mTORC2 SIN1_3R) (C) Surface representation of mTORC2. SIN1 (shown as green cartoon) interacts via two N-terminal helices with Rictor, winds around Rictor, traverses the catalytic site cleft and winds around mLST8. The field of view of subpanel C is indicated. (D) Close-up view of the SIN1 N-terminal residues, which are deeply inserted between Rictor AD and HD. Acetylated Ala2 and Phe3 are bound in a hydrophobic pocket, while Asp5 interacts via salt bridges (yellow dashes). (E) Top view of mLST8 $\beta$-propeller (orange) and the interaction regions with SIN1 (green). The nomenclature for WD40 $\beta$-propeller repeats is indicated. (F) Top view of the catalytic site with the structure shown as surface together with the density of a subclass (light grey). The lower resolution extra density is consistent with a placement of the SIN1 CRIM domain, here shown in dark green (PDB: 2RVK). Unassigned extra density protrudes from the CRIM domain to the mTOR active site and Rictor. 
A

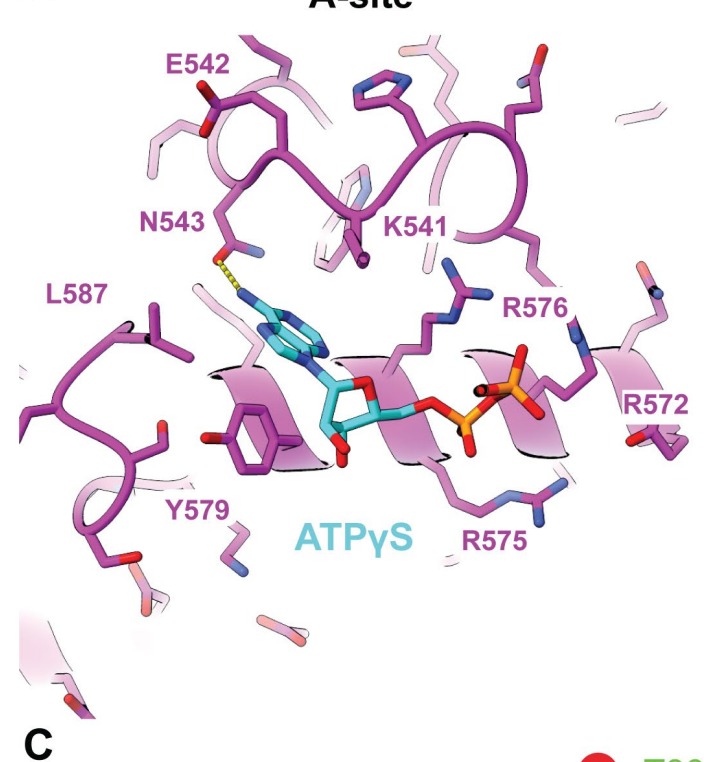

B

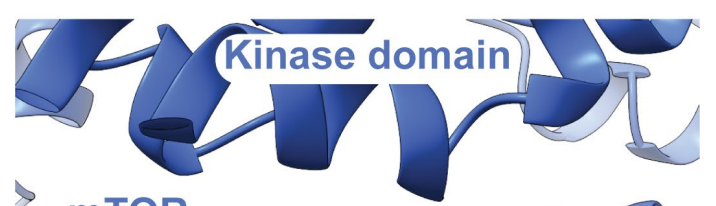

$>\mathrm{mTOR}$

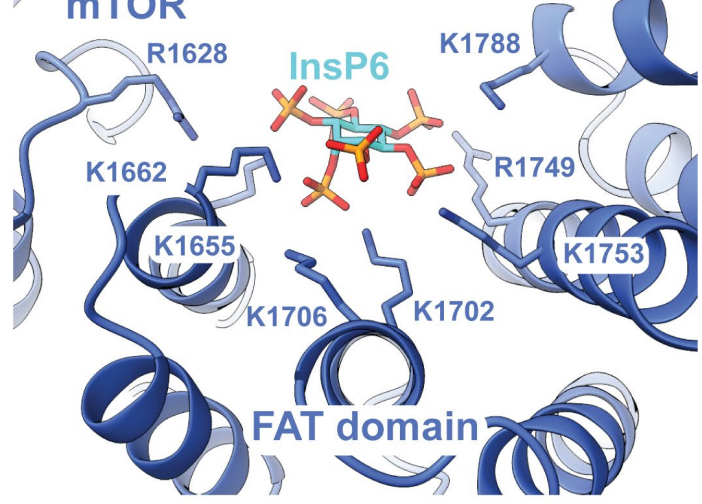

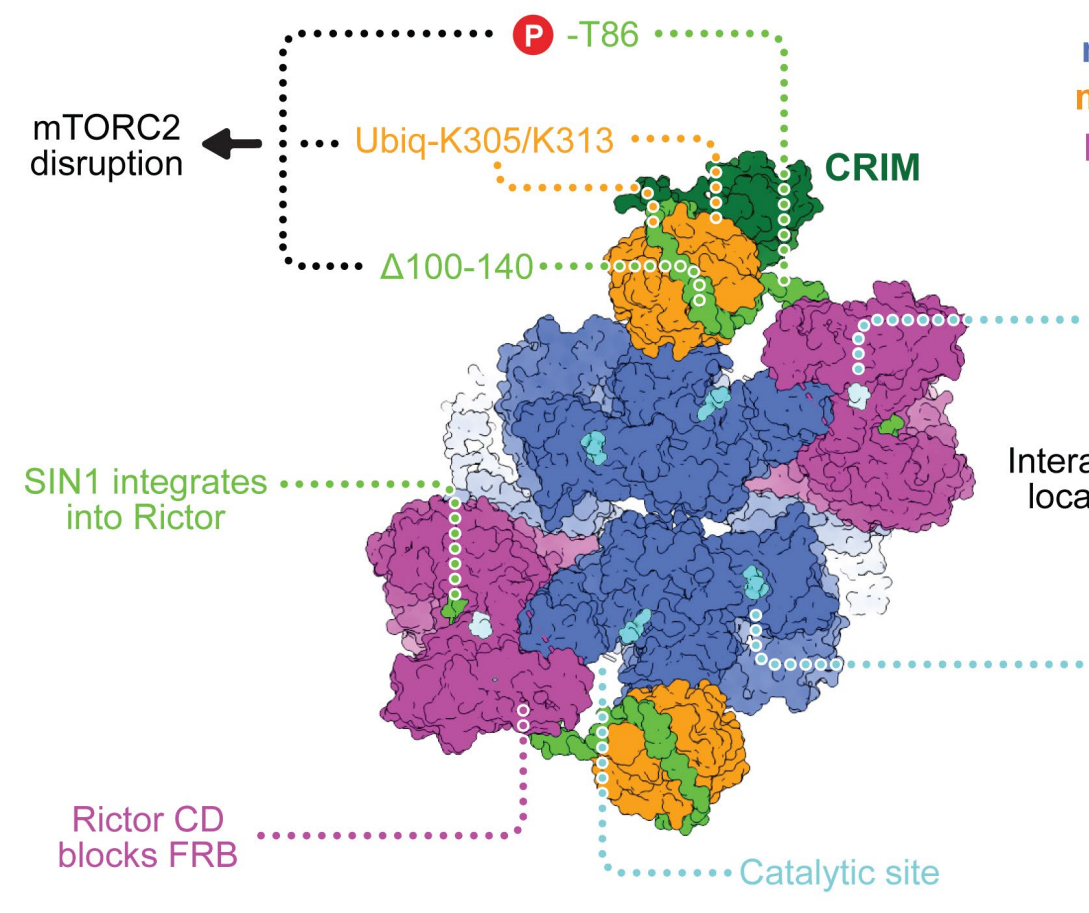

mTOR

mLST8

Rictor

SIN1

A-site

mTORC2

$\downarrow \ominus \oplus$

interactions affecting

localization and/or

regulation

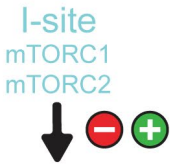

Folding

and/or

Assembly

Fig. 4. Small molecule binding sites of mTORC2 outside the active site region (A). Close-up view of the A-site on the periphery of the Rictor HD with bound ATP $\gamma \mathrm{S}$. A hydrogen bond between ATP $\gamma \mathrm{S}$ and Asn543is shown as dashed yellow lines. (B) Close-up view of the I-site in the FAT domain of mTOR. InsP6 is surrounded by a cluster of positively charged amino acids. It only directly interacts with residues of the FAT domain. (C) Overview of mTORC2 architecture and 
ligand interaction sites. Each half of the dimeric mTORC2 has three small molecule binding sites. The kinase active site and the A-site, which is located in the peripheral region of Rictor, bind to ATP (or ATP analogues). The I-site in the middle of the FAT domain of mTOR binds InsP6. The indicated modifications on SIN1 and mLST8 affect mTORC2 assembly. Extra-density region following the CRIM domain is indicated as a grey outline. 